ultrasound measurement. II. Clinical application of a new formula. Acta Obstet Gynecol Scand 1982;61:307-12.

10 Eik-Nes SH, Maršál K, Kristoffersen K. Methodology and basic problems related to blood flow studies in the human fetus. Ultrasound Med Biol 1984;10:329-37.

11 Lingman G, Maršal K. Fetal central blood circulation in the third trimester of normal pregnancy. Longitudinal study. II. Aortic blood velocity waveform. Early Hum Dev 1986;13:151-9.

12 Gudmundsson S, Maršal K. Umbilical artery and uteroplacental blood flow velocity waveforms in normal pregnancy-a cross-sectional study. Acta Obstet Gynecol Scand 1988;67:347-54.

13 Man In'T Veld AJ, Schalekamp MADH. How intrinsic sympathomimetic activity modulates the haemodynamic responses to $\beta$-adrenoceptor activity modulates the haemodynamic responses to $\beta$-adrenoceptor
antagonists. A clue to the nature of their antihypertensive mechanism. antagonists. A clue to the nature of their a
14 Thulesius $\mathrm{O}$, Gjöres JE, Berlin E. Vasodilating properties of beta-adrenoceptor blockers with intrinsic sympathomimetic activity. Br $\mathrm{f}$ Clin Pharmaco 1982;13:S229-30.

15 Clark BJ. Pharmacological analysis of the intrinsic sympathomimetic activity of pindolol: evidence for selective beta-2-adrenoceptor stimulation. Triangle 1984;23:33-8.

16 Lundgren Y, Karlsson K, Ljungblad U. Acute hemodynamic effects of pindolol during pregnancy in experimental renal hypertension. Acta Obstet Gynecol Scand 1984;Suppl 118:S85-90.

17 Duckles SP, Banner W Jr. Changes in vascular smooth muscle reactivity during development. Ann Rev Pharmacol Toxicol 1984;24:65-83.

18 Butters L, Kennedy S, Rubin PC. Atenolol in essential hypertension during pregnancy. $B M \mathcal{F}$ 1990;301:587-9.

(Accepted 21 fanuary 1992)

\title{
Review of management of incidents involving exposure to blood in a London teaching hospital, 1989-91
}

\author{
Katie Oakley, Christopher Gooch, Anne Cockcroft
}

\begin{abstract}
Objective-To review management of incidents involving exposure to blood reported to an occupational health unit.

Design-Analysis of all reported incidents from January 1989 to June 1991.

Setting-London teaching hospital.

Subjects -447 health care workers and students.

Main outcome measures-Immunisation against hepatitis $B$ virus before exposure, proportion of known source patients tested for hepatitis B surface antigen and HIV antibodies, and reasons for not testing known source patients.
\end{abstract}

Results -447 incidents were reported: 337 sharps injuries and 110 other exposures. 310 staff reporting incidents $(205(82 \%)$ nurses) were already immune to hepatitis B virus, nearly always because of immunisation. 345 source patients were identified, 77 of whom had already been tested for hepatitis $B$ surface antigen (28 positive results) and 58 for HIV antibodies (18 positive results). Of those not previously tested, 145 of 266 were subsequently tested for hepatitis B surface antigen (two positive) and 149 of 287 for HIV antibodies (none positive). The main reasons for not testing source patients were that the incident was not considered a risk, that the patient had gone home, and that the clinical team were unwilling to ask the patient. Specific hepatitis B immunoglobulin was given to 18 staff who were not immune and was avoided in 11 cases by a negative result for the patient. Prophylactic zidovudine was discussed but not given to any staff member.

Conclusions-Management of exposure to blood is improved by widespread immunisation against hepatitis $B$ virus and by knowledge of source patients' hepatitis B virus and HIV status.

Occupational Health Unit, Royal Free Hampstead NHS Trust and Royal Free Hospital School of Medicine, London NW3 20G

Katie Oakley, senior nurse Christopher Gooch, statistician

Anne Cockcroft, senior lecturer in occupational medicine

Correspondence to:

Dr Cockcroft.

BMF 1992;304:949-51

\section{Introduction}

Sharps injuries and other exposure to patients' blood carry a risk of transmission of bloodborne infections such as hepatitis B virus and HIV. Protection of staff requires immunisation against hepatitis $B$ virus, efforts to reduce the number of incidents, an effective reporting system, and proper management of reported incidents.

Since January 1989 when staff have been exposed to patients' blood our hospital has attempted to test the source patient for hepatitis B surface antigen and antibodies to HIV after counselling and consent, as is officially recommended in both Britain and the United States. ${ }^{2-4}$ Staff are encouraged to report incidents to our occupational health department and we contact the clinical team caring for the source patient asking them to approach the patient for the tests. Staff not immune to hepatitis B virus are given specific hepatitis B immunoglobulin if the source patient is positive for hepatitis B surface antigen. Prophylactic zidovudine is considered for exposures to blood containing HIV antibodies. We reviewed all exposures to blood reported to our unit during the two and a half years from January 1989 to June 1991.

\section{Methods}

Details of all reported incidents were recorded on a standard form which included the hepatitis B immunisation status and occupational group of the staff member, the hepatitis B virus and HIV status of the source patient, and whether the patient was tested as a result of the incident. Reasons for not testing known source patients were recorded.

One way frequency distributions were produced for each variable and relations between variables were analysed by generating two way frequency distributions. Overall relations between variables were assessed using Pearson's $\chi^{2}$ test for contingency. Percentage estimates and their confidence intervals were derived to compare particular categories within each of the variables.

\section{Results}

A total of 447 incidents was reported, 337 of which were incidents with needles or sharp instruments; there were 110 splash, bite, or scratch incidents. In 345 cases the source patient was known. Some patients had already been tested for hepatitis B surface antigen and HIV antibodies before the incident. One hundred and forty nine were tested for HIV antibodies and 145 for hepatitis B surface antigen because of the incident (table I). Eighteen of the patients were HIV positive; all had been tested before the incident. Thirty patients

TABLE I-Numbers of known source patients in blood exposure incidents tested for hepatitis $B$ surface antigen and HIV antibodies

\begin{tabular}{lcccc}
\hline & \multicolumn{4}{c}{ Tested for hepatitis B surface antigen } \\
\cline { 2 - 5 } $\begin{array}{l}\text { Tested for HIV } \\
\text { antibodies }\end{array}$ & $\begin{array}{c}\text { Before } \\
\text { incident }\end{array}$ & $\begin{array}{c}\text { After } \\
\text { incident }\end{array}$ & $\begin{array}{c}\text { Not } \\
\text { tested }\end{array}$ & Total \\
\hline $\begin{array}{lcccc}\text { Before incident } \\
\text { After incident }\end{array}$ & 48 & 4 & 4 & $58^{\star}$ \\
Not tested & 13 & 129 & 7 & 149 \\
\hline Total & 16 & 12 & 110 & 138 \\
\hline
\end{tabular}

$\star$ In two cases we could not determine whether the patient was tested for hepatitis B surface antigen. 
were positive for hepatitis B surface antigen; 28 had been tested before the incident and two were found to be positive when tested because of the incident.

Nurses reported most of the incidents (256), with 76 being reported by student nurses. Doctors and medical students accounted for a further 82 reported incidents: 44 were reported by medical doctors, 24 by surgeons, and 14 by medical students. Other staff reported 109 injuries.

There was significant variation in the type of incident by job category $\left(\chi^{2}=16 \cdot 37, \mathrm{df}=4 ; \mathrm{p}<0.005\right)$, with nurses sustaining proportionally fewer sharps injuries than other groups. The proportions of reported incidents that were sharps injuries differed between doctors $(73,89 \%)$ and nurses $(179,70 \%)$ by $19 \%$ (95\% confidence interval $10 \cdot 3 \%$ to $27 \cdot 9 \%$ ) and between ancillary staff $(45,87 \%)$ and nurses by $17 \%$ $(5 \cdot 8 \%$ to $27 \cdot 5 \%)$.

Immunity was not determined in nine staff. Of the remaining $438,310(71 \%)$ staff had immunity to hepatitis B virus. Nearly all of these (304) had received all or part of an immunisation course; $a$ further six were positive for hepatitis $B$ core antibody, reflecting previous infection. Of the 128 staff who were not immune, 19 had not seroconverted after immunisation, 24 were part way through an immunisation course, 83 had not been immunised, and in two it was not known whether they had been immunised. Immune status varied significantly with occupational category $\left(\chi^{2}=46.5\right.$, $\mathrm{df}=4 ; \mathrm{p}<0.001$ ) (table II). The proportions immune differed between nurses and doctors by $13 \%(1.7 \%$ to $24 \cdot 2 \%)$ and between nurses and ancillary staff by $32 \%$ $(17 \cdot 3 \%$ to $46 \cdot 1 \%)$.

TABLE II-Immunity to hepatitis B virus among 438 hospital staff exposed to patients' blood

\begin{tabular}{lcc}
\hline & $\begin{array}{c}\text { No (\%) } \\
\text { immune to } \\
\text { hepatitis B virus }\end{array}$ & $\begin{array}{c}95 \% \text { confidence } \\
\text { interval }\end{array}$ \\
\hline Nurses $(\mathrm{n}=251)$ & $205(82)$ & 193 to 217 \\
Doctors $(\mathrm{n}=80)$ & $55(69)$ & 46 to 63 \\
Ancillary workers $(\mathrm{n}=52)$ & $26(50)$ & 19 to 33 \\
Paramedics $(\mathrm{n}=18)$ & $10(56)$ & 6 to 14 \\
Others $(\mathrm{n}=37)$ & $14(38)$ & 8 to 20 \\
\hline
\end{tabular}

In all, 138 known source patients were not tested for HIV antibodies and 121 not tested for hepatitis B surface antigen. Source patients were usually tested either for both HIV antibody and hepatitis B surface antigen or for neither (table I). The most common reason for not testing the source patient was that the incident was assessed as not posing a risk of infection (42 cases). The next most common reason was that the source patient had gone home ( 31 cases). In other cases either the clinical team ( 25 cases) or the affected staff member ( 21 cases) did not want the source patient tested. Seventeen of 166 source patients who were approached refused consent for testing.

Eighteen staff who were not immune were given specific hepatitis B immunoglobulin after incidents with blood from patients positive for hepatitis $B$ virus or with needles throught to have been used on such patients. Administration of the immunoglobin was avoided in 11 staff members by the timely finding of a negative result for the source patients. In 28 of the cases involving staff who were not immune the patient was not tested for hepatitis B surface antigen.

None of the staff in the 18 reported incidents with source patients positive for HIV antibody was given prophylactic zidovudine. Some of the incidents were considered not to pose an infection risk. In the remainder after discussion the staff members chose not to take zidovudine. Follow up HIV antibody tests on the staff concerned have given negative results so far.

\section{Discussion}

In our study $71 \%$ of staff reporting exposure to patients' blood were immune to hepatitis B virus. This compares with rates of $25 \%,{ }^{5} 73 \%,{ }^{6}$ and $65 \%{ }^{7}$ reported in other studies. This immunisation coverage is not necessarily equivalent to the coverage in the whole population of hospital staff; we are currently investigating this.

We do not know the actual number of blood exposure incidents that occurred during the study period, but other studies have emphasised the underreporting of contamination incidents, especially among doctors. ${ }^{8}$ The higher proportion of sharps incidents reported by doctors compared with nurses may be because doctors are more likely to report sharps injuries than other incidents. We are trying to improve reporting rates: we have established a special telephone number for reporting incidents, medical students and junior doctors are given talks and written information about reporting, and we have been surveying all blood exposures in operating theatres and will feedback the results to the staff concerned. High reporting rates are important because reported incidents inform our continuing efforts to reduce incidents by changing practices and introducing safer equipment.

The main benefit of testing known source patients was the value of negative results. We were able to avoid giving hepatitis B immunoglobulin to several staff and to reassure most staff about the risk of HIV transmission from the incident. Many staff are extremely worried after incidents and are relieved to know the patient's HIV antibody status. No staff member has so far chosen to take prophylactic zidovudine. The value of zidovudine after needlestick injuries from HIV positive patients is unproved, ${ }^{10}$ and its failure to prevent seroconversion has been documented. ${ }^{11-13}$

Testing for hepatitis B surface antigen and HIV antibodies was undertaken in only about half of eligible source patients. The main problem was one of logistics; it proved difficult to test patients who had gone home when the incident was reported. A study from the United States also reported logistical problems in testing source patients. ${ }^{14} \mathrm{We}$ have tried to organise testing at subsequent outpatient visits, but this often does not work. For incidents that occur in the community patients are visited by an occupational health nurse together with the district nurse after liaison with the general practitioner.

In the early months of this study clinical teams were often unwilling to approach their patients, especially for HIV testing. We tackled this problem by discussions with senior clinicians and by running training sessions for medical students and junior doctors and providing material to help them approach patients. Counselling before HIV testing can and should be undertaken by other health care workers as well as by specialist counsellors. ${ }^{15} 16$

It is debatable whether clinicians should have the right to veto their patients being approached for testing after blood exposure incidents. The rights of the staff member have to be considered as well as the possible adverse effects on the patient. The patient, once approached, has the option to refuse, although in this study few did so.

For most of the incidents reported during this study it was possible to advise and treat the staff members based on information about themselves and the source patients. We need to increase the proportion of source patients tested for blood borne viruses after incidents and to increase further the proportion of staff immunised against hepatitis $B$ virus.

We thank the staff of the occupational health unit, the virology department, and the AIDS counselling team. KO 
was funded by North East Thames Regional Health Authority.

1 British Medical Association. A code of practice for the safe use and disposal of sharps. London: BMA, 1990.

2 Advisory Committee on Dangerous Pathogens. HIV-the causative agent of AIDS and related conditions. 2nd ed. London: HMSO, 1990.

3 Department of Health Expert Advisory Group on AIDS. Guidance for clinical health care workers: protection against infection with HIV and hepatitis viruses. London: HMSO, 1990.

4 Centrs for Disease Control. Guidelines for prevention of transmission of human immunodeficiency virus and hepatitis B virus to health care and public safety workers. $M M W R$ 1989;38.S 6

Yassi A, McGill M, Jeanson S, Sekla L. Anti HBs protection in hospital workers vaccinated against hepatitis $B$ : policy implications of a pilot study. Br f Indust Med 1990;47:645-8.

6 Porteous MJ LeF. Operating practices and precautions taken by orthopaedic surgeons to avoid infection with HIV and hepatitis B virus during surgery.

7 Burden AD, Whorwell PJ. Poor uptake of hepatitis B immunisation amongs hospital-based health care staff. Postgrad Med F 1991;67:256-8.

8 Astbury C, Baxter PJ. Infection risks in hospital staff from blood: hazardous iniury rates and acceptance of hepatitis B immunisation. $\mathcal{F}$ Soc Occup Med $1990 \cdot 40 \cdot 92-3$

9 Jeffries DJ. Zidovudine after occupational exposure to HIV. BMF 1991;302: 1349-51.

10 Brown EM, Caul EO, Roome APCG, Glover SC, Reeves DS, Harling CC. Zidovudine after occupational exposure to HIV. BMJ 1991;303:990

11 Lange JMA, Boucher CAB, Hollak CEM, Wiltink EHH, Reiss P, Van Royen $\mathrm{EA}$, et al. Failure of zidovudine prophylaxis after accidental exposure to HIV-1. N Engl F Med 1990;322:1375-7.

12 Looke DFM, Grove DI. Failed prophylactic zidovudine after needlestick injury. Lancet 1990;335:1280.

13 Tait DR, Pudifin DJ, Gathiram V, Windsor IM. Zidovudine after occupational exposure to HIV. BMf 1991;303:581.

14 Miller PJ, Farr BM. A study of the rate of postexposure human immunodeficiency virus testing in a hospital requiring written informed consent. fOccup Med 1989;31:524-7.

15 Miller R, Bor R. AIDS: a guide to clinical counselling. London: Science Press,

16 Bor R, Miller R, Johnson M. A testing time for doctors: counselling patients before an HIV test. $B M F$ 1991;303:905-7.

(Accepted 13 February 1992)
Childhood Cancer

Research Group,

University of Oxford,

Oxford OX2 6HJ

M M Hawkins,

epidemiologist

L M Kinnier Wilson, medical research officer

M H N Potok, computer

programmer

M D Anderson Cancer Center, University of Texas, USA

M A Stovall, radiation physicist

Royal Manchester Children's Hospital, Pendlebury, Manchester H B Marsden, professor of paediatric pathology

St Bartholomew's Hospital, London EC1

$\mathrm{J}$ E Kingston, consultant paediatric oncologist

Hospital for Sick Children, Great Ormond Street, London

$\mathrm{J} M$ Chessells, professor of

haematology and oncology

Correspondence to: $\mathrm{Dr}$ Hawkins.

BMJ 1992;304:951-8

\title{
Epipodophyllotoxins, alkylating agents, and radiation and risk of secondary leukaemia after childhood cancer
}

\author{
M M Hawkins, L M Kinnier Wilson, M A Stovall, H B Marsden, M H N Potok, J E Kingston, \\ J M Chessells
}

\section{Abstract}

Objective-To investigate the incidence and aetiology of secondary leukaemia after childhood cancer in Britain.

Design-Cohort study and a case-control study.

Setting-Britain and population based National Register of Childhood Tumours.

Subjects-Cohort of 16422 one year survivors of childhood cancer diagnosed in Britain between 1962 and 1983, among whom 22 secondary leukaemias were observed. A case-control study of 26 secondary leukaemias observed among survivors of childhood cancer diagnosed in Britain between 1940 and 1983; 96 controls were selected matched for sex, type of first cancer, age at first cancer, and interval to diagnosis of secondary leukaemia.

Main outcome measures-Dose of radiation averaged over patients' active bone marrow and total accumulated dose of epipodophyllotoxins, alkylating agents, vinca alkaloids, antimetabolites, and antibiotics $\left(\mathrm{mg} / \mathrm{m}^{2}\right)$ given for the original cancer.

Results - Cumulative risk of secondary leukaemia within the cohort did not exceed $0.5 \%$ over the initial five years beyond one year survival, except that after non-Hodgkin's lymphomas $1.4 \%$ of patients developed secondary leukaemia. Corresponding figure for patients treated for non-Hodgkin's lymphomas in the early 1980 s was $4 \%$. The relative risk of secondary leukaemia increased significantly with exposure to epipodophyllotoxins and dose of radiation averaged over patients' active bone marrow. Ten patients developed leukaemia after having an epipodophyllotoxin-teniposide in nine cases, etoposide in one. Chromosomal translocations involving 11q23 were observed relating to two secondary leukaemias from a total of six for which there were successful cytogenetic studies after administration of an epipodophyllotoxin.

Conclusions-Epipodophyllotoxins acting alone or together with alkylating agents or radiation seem to be involved in secondary leukaemia after childhood cancer.

\section{Introduction}

An excess of leukaemia has been observed after treatment of a variety of adult ${ }^{1-6}$ and childhood cancers. ${ }^{78}$ Case-control studies have identified specific groups of cytotoxic drugs that are associated with an increased risk of secondary leukaemias ${ }^{9-12}$; these and other studies have particularly identified alkylating agents as being associated with an increased risk of secondary leukaemia after adult and childhood cancers. Radiation exposure is undoubtedly involved in some leukaemias. ${ }^{13}$ There have also been suggestions that the epipodophyllotoxins may be related to an increased risk of subsequent leukaemias. ${ }^{14-21}$ We report the results of a population based cohort study and a casecontrol study carried out $(a)$ to estimate the absolute risk of secondary leukaemia after childhood cancer in Britain, and $(b)$ to determine those aspects of treatment related to an increased relative risk of secondary leukaemia.

\section{Methods}

PATHOLOGICAL CONFIRMATION OF NEOPLASMS

Each case included in the case-control study had representative slides of both the first and second neoplasms centrally reviewed and confirmed by a paediatric histopathologist (HBM). In addition, each possible occurrence of leukaemia after an initial leukaemia or non-Hodgkin's lymphoma was centrally reviewed by a haematologist (JMC). For each control we obtained the definitive pathology report(s) confirming the diagnosis concerned. For those patients included in the cohort study who did not develop a secondary leukaemia almost all diagnoses were histologically verified except for $4 \%$ of the leukaemias, $9 \%$ of the central nervous system tumours, and $8 \%$ of the retinoblastomas, which were based on blood counts, radiology or scans, and observation under anaesthesia respectively.

\section{COHORT STUDY}

From the population based National Register of Childhood Tumours maintained by the Childhood Cancer Research Group we identified children aged under 15 years who were diagnosed with cancer between 1962 and 1983 while resident in Britain and who subsequently survived at least one year. Entry to the study occurred one year after diagnosis, and patients exited when the first of one of the following occurred: a secondary leukaemia was diagnosed; the 